\title{
Risk factors affecting outcome and morbidity in the surgical management of bronchiectasis
}

Sevval Eren, MD, ${ }^{a}$ Hidir Esme, MD, ${ }^{b}$ and Alper Avci, $M^{a}{ }^{a}$

Earn CME credits at http://cme.ctsnetjournals.org

Objective: Bronchiectasis continues to be a major cause of morbidity and mortality in developing countries. The purpose of this study was to present the results of our 14 years of surgical experience to re-evaluate our indications for using surgical therapy and to analyze several factors that might affect the outcome and postoperative complications of this disease.

Method: Age, sex, etiologic factors, symptoms, the duration of symptoms, radiologic and radionuclide examinations, preoperative evaluation, surgical procedures, postoperative morbidity and mortality, and the follow-up results from 143 patients operated on for bronchiectasis between January 1992 and January 2006, were reviewed retrospectively.

Results: One hundred forty-three patients underwent 148 operations for bronchiectasis. The mean age was 23.4 years. Complete resection was achieved in 118 patients. The morbidity rate was $23.0 \%$ and the mortality rate was $1.3 \%$. Postoperatively, $75.9 \%$ of the patients were free of symptoms, $15.7 \%$ were improved, and $8.2 \%$ showed no improvement or were worse. The logistic regression analysis showed that a history of tuberculosis and incomplete resection were independent predictors of the operative result. Moreover, the lack of a preoperative bronchoscopic examination, a forced expiratory volume in 1 second of less than $60 \%$ of the predicted value, a history of tuberculosis, and incomplete resection were independent predictors of postoperative complications.

Conclusions: A history of tuberculosis and incomplete resection were risk factors both for postoperative complications and for a worse operative result. The lack of a preoperative bronchoscopic examination and a low forced expiratory volume in 1 second were risk factors for postoperative complications. Surgery for multiple segments on different lobes should be performed whenever possible.

From the Department of Thoracic Surgery, Dicle University, School of Medicine, Diyarbakir, Turkey; and the Department of Thoracic Surgery, ${ }^{\mathrm{b}}$ Kocatepe University, School of Medicine, Afyon, Turkey.

Received for publication Dec 15, 2006; revisions received April 2, 2007; accepted for publication April 11, 2007.

Address for reprints: Sevval Eren, MD, Dicle University School of Medicine, Department of Thoracic Surgery, 21280 Diyarbakir, Turkey (E-mail: sevval@dicle.edu.tr).

J Thorac Cardiovasc Surg 2007;134:392-8

$0022-5223 / \$ 32.00$

Copyright (C) 2007 by The American Association for Thoracic Surgery

doi:10.1016/j.jtcvs.2007.04.024
$\mathrm{T}$ he term bronchiectasis is derived from the Greek bronchus and ektasis, meaning dilatation. Bronchiectasis was first described by Laenec in 1819 and, before the antibiotic era, was considered a morbid disease with a high mortality rate from respiratory failure and cor pulmonale. ${ }^{1}$ With the development of antibiotics in the 1940s, this entity began to be seen less frequently, but, with the emergence of drug-resistant microorganisms and the increasing frequency of drugresistant tuberculosis, an increased incidence of postinfectious bronchiectasis is being noted. ${ }^{2}$ The current increase in tuberculosis rates is directly related to insufficient and irregular medication. Additionally, irregular and inadequate treatment, the cessation of medication shortly after symptom improvement, and a lack of check-ups after treatment are factors accelerating recurrent pulmonary infection in developing countries. Therefore, bronchiectasis continues to be a major cause of morbidity and mortality in developing countries as well as in Turkey. ${ }^{3-5}$

Complications of this condition include hemoptysis, pulmonary abscess, brain abscess, amyloidosis, and respiratory failure. Cor pulmonale accounts for $37 \%$ of 


\section{Abbreviations and Acronyms}

HRCT $=$ high-resolution computed tomography

$\mathrm{SaO}_{2}=$ arterial oxygen saturation

deaths. ${ }^{6}$ However, risk factors that may affect the outcome and morbidity in patients with bronchiectasis have not been elucidated fully. The purpose of this study was to present the results of our 14 years of surgical experience to reevaluate our indications for using surgical therapy and to analyze several factors that might affect the outcome and postoperative complications of this disease.

\section{Patients and Methods}

We reviewed the medical records of 143 patients who underwent surgical resection for bronchiectasis between January 1992 and January 2006 at the thoracic surgery departments of Dicle and Kocatepe Universities. The following variables were analyzed: age, sex, history of tuberculosis, symptoms, duration of symptoms, radiologic and radionuclide examinations, results of bacterial cultures of sputum and/or bronchial aspirate and preoperative pulmonary function test or exercise-tolerance test, whether studies with rigid and/or flexible bronchoscopes had been performed, type of bronchiectasis, type of operation, postoperative morbidity and mortality, the result of surgical therapy, and the follow-up results.

Patients were chosen as candidates for surgical treatment according to the following criteria: localized bronchiectasis documented by high-resolution computed tomography (HRCT); adequate cardiopulmonary reserve; symptoms such as chronic productive cough, repeated or significant hemoptysis, lung abscess, empyema, and recurrent pulmonary infections; and failure of medical treatment. Medical therapy constituted judicious use of systemic antibiotics based on current sputum or bronchoscopic lavage cultures, mucolytic agents, nonirritant expectorants, postural drainage, humidification, anti-inflammatory agents, and bronchodilators. Failure of medical treatment was defined as frequent exacerbations interfering with normal professional or social life or requiring multiple hospitalizations. The locus of hemorrhage was identified bronchoscopically in patients with repeated or significant hemoptysis, and, after the lungs were assessed by HRCT, surgery was planned in patients who did not respond well enough to conservative treatment.

The initial diagnosis was based on the history and physical examination. The evaluation process included radiologic and physiologic parameters. Radiologic information included chest radiographs for all patients. Before 1996, preoperative bronchography and/or chest computed tomography was performed to evaluate the severity and distribution of bronchiectasis. Since 1996, HRCT has supplanted bronchography in the diagnosis of bronchiectasis. Pulmonary function tests were performed routinely in older children and all adult patients. Children younger than 10 years old were evaluated by an exercise-tolerance test. Pulmonary ventilation/ perfusion scan was performed to estimate the postoperative lung function, and appropriate cardiorespiratory reserve was necessary for operative consideration. Laboratory tests were also run to determine cardiac, renal, and hepatic functions. Examination with a rigid and/or flexible bronchoscope was also performed in 117 $(81.8 \%)$ patients for the removal of secretions and for determining foreign body or endobronchial lesions. The bronchial aspirate and sputum were cultured routinely for pyogenic and tubercular bacilli.

All patients received intensive chest physiotherapy during the preoperative period. Patients were given antibiotics on the basis of the sputum and/or bronchial aspirate culture. For patients reporting sputum production, chest physiotherapy and expectorant and preoperative antimicrobial therapy were continued until the daily volume of sputum decreased to a minimum. Prophylactic antibiotics were given for 48 hours before the operation in patients with negative culture antibiograms. With the intention of timing the operation when tuberculosis was inactive, we performed elective surgery usually 6 months after the last positive sputum culture for acid-fast bacilli and after the completion of antituberculosis treatment.

Posterolateral thoracotomy was performed in all patients. Thoracotomy was performed so as to conserve as much muscle as possible. A left-sided double-lumen endotracheal tube was used to avoid contralateral contamination of secretions. In children under 13 years old, in whom the double-lumen endotracheal tube was not used, bronchoscopy was performed, and the bronchus of the side ready for resection was cleaned by aspiration before the introduction of an endotracheal tube. A Fogarty embolectomy catheter was used as a bronchus blocker in 3 patients, with satisfactory results.

Excessive bronchial dissection was avoided, and peribronchial tissues were preserved. The bronchial stump was manually sutured with nonabsorbable materials in some patients or closed with a mechanical stapler in others, and a stump covering with mediastinal pleura or tissue was performed. All specimens had pathologic confirmation of bronchiectasis. Complete resection is defined as an anatomic resection of all affected segments assessed preoperatively by either HRCT or bronchography. Postoperative complications were monitored for 30 days. Operative mortality was defined as a patient's death within 30 days of thoracotomy.

At the final follow-up, after 6 months, the outcome of surgery was evaluated according to the following criteria: excellent (complete absence of preoperative symptoms that led to surgery), good (marked reduction in preoperative symptoms but needing antibiotic therapy occasionally), no change (no reduction in preoperative symptoms, and no decrease in hospital admissions or medical therapy requirements), and worse (frequent exacerbations of disease requiring hospitalization).

Clinical data were calculated by the mean and standard deviation. The influences of some variables on the prognosis after the operation were studied by dividing the patients into an improved group ("excellent" or "good" outcomes) and an unimproved group ("no change" or "worse" outcomes). Logistic regression analyses were used to compare a variety of clinical factors between the groups. Additionally, the association between potential risk factors and postoperative complication was calculated.

\section{Results}

The mean age of the 143 patients at the time of thoracotomy was 23.4 years (range, 3-63 years). There were 52 female and 91 male patients. Thirty-nine $(27.2 \%)$ patients were less than 17 years old. The mean duration of the symptoms was 28.5 months (range, 0-156 months). One hundred thirteen $(79.0 \%)$ 
patients had chronic symptoms present for a mean of 47.4 months (range, 12-156 months). There was a history of tuberculosis in $22(15.3 \%)$ patients. Presenting symptoms of the patients were summarized in Table 1 .

Preoperative diagnostic studies included chest radiographs in all patients, HRCT in 104 (72.7\%), contrast bronchography in $26(18.1 \%)$, computed tomography in 19 (13.2\%), and bronchoscopy in 117 (81.8\%). Bronchoscopy was not performed in 26 patients. Eight of them refused bronchoscopy. The other 18 were pediatric patients, and we did not have a pediatric bronchoscope available for ages 5 to 10 between 1992 and 1996. We have been performing bronchoscopy on all pediatric and adult patients preoperatively for 10 years. The type of bronchiectasis was saccular in $66(46.1 \%)$ patients, cylindrical in 47 (32.8\%), and varicose in $20(13.9 \%)$. The disease was unilateral in 132 $(92.3 \%)$ patients and bilateral in $11(7.6 \%)$. The mean number of segments involved was 3.4 (range, 1-12 segments). Four or fewer segments were involved in 76 $(53.1 \%)$.

One hundred thirty-four (93.7\%) patients had respiratory function tests, with the majority $(79.1 \%)$ showing normal ventilatory patterns. The remainder showed a mixed or obstructive ventilatory pattern. The 6-minute walk test was performed along a level hospital corridor in 9 younger children. Arterial oxygen saturation $\left(\mathrm{SaO}_{2}\right)$ was measured by finger pulse oximetry before and during the test and monitored continuously. Measurements were recorded at 30second intervals. All patients were able to walk continuously for the 6-minute period. The lowest $\mathrm{SaO}_{2}$ was used to calculate minimum $\mathrm{SaO}_{2}$. Mean minimum $\mathrm{SaO}_{2}$ was 94.7. Care was taken to ensure that $\mathrm{SaO}_{2}$ did not decrease below $90 \%$. Along with exercise, a $2 \%$ or greater desaturation was considered to represent a risk. ${ }^{7}$ One patient seen to be at risk received 3 weeks of additional chest physiotherapy, incentive spirometry, and ambulation with physical therapy. The patient underwent surgery after this course of treatment. No patient was observed to have effort dyspnea after the test.

The most common microorganism that was obtained from preoperative sputum cultures was Haemophilus influenzae (Table 2). The most common probable etiology was postobstructive pneumonitis (Table 3 ). The indication for

TABLE 1. Presenting symptoms of the patients

\begin{tabular}{lrr}
\hline Symptom & $\mathbf{n}$ & $\%$ \\
\hline Productive cough & 94 & 65.7 \\
Fetid sputum & 72 & 50.3 \\
Recurrent infections & 69 & 48.2 \\
Hemoptysis & 21 & 14.6 \\
Chest pain & 12 & 8.3 \\
Fever & 5 & 3.4 \\
No symptom & 2 & 2.0 \\
\hline
\end{tabular}

TABLE 2. Microbiologic culture results of the patients

\begin{tabular}{lrr}
\hline Microorganism & $\mathbf{n}$ & $\%$ \\
\hline Haemophilus influenzae & 18 & 12.5 \\
Staphylococcus aureus & 13 & 9.0 \\
Streptococcus pneumoniae & 9 & 6.2 \\
Pseudomonas aeruginosa & 8 & 5.5 \\
Bacteroides and other anaerobes & 7 & 4.8 \\
Klebsiella pneumoniae & 5 & 3.4 \\
Negative culture & 83 & 58.0 \\
\hline
\end{tabular}

pulmonary resection was failure of medical therapy in 106 $(74.1 \%)$ patients, recurrent or massive hemoptysis in 21 $(14.6 \%)$, lung abscess in $9(6.2 \%)$, and empyema in 7 $(4.8 \%)$.

Of the 21 patients with with hemoptysis, 4 had massive hemoptysis. These 4 patients underwent surgery after bronchoscopic evaluation and intervention. Massive hemoptysis was defined as expectorating more than $600 \mathrm{~mL}$ of blood in 24 hours. A rigid broncoscope was used under emergency conditions to determine the location of hemorrhage in patients with massive hemoptysis. Irrigation with physiologic saline with ice was performed to stop the bleeding. Then the bleeding bronchus was catheterized for 1 hour via a doublelumen endotracheal tube. In 3 of these 4 patients, the locus of hemorrhage was clearly detected; in the fourth the hemorrhage could not be exactly localized. HRCT revealed signs of destroyed lung in 2 patients who required left pneumonectomy. Among 17 patients with repeated or significant hemoptysis, there was unilateral hemorrhage in 15 , whereas the locus of hemorrhage was bilateral in the remaining 2. Once hemorrhage was under control, these patients underwent resection. Six (4.1\%) patients had undergone prior operations for bronchiectasis: 4 had had lobectomy and 2 lobectomy and lingulectomy.

One hundred forty-three patients underwent 148 operations for bronchiectasis. Two patients underwent staged thoracotomies. Rethoracotomy owing to complications was performed in 3 patients. The types of procedures are presented in Table 4. Six of the patients undergoing pneumonectomy had completion pneumonectomies. Eight of the patients who had pneumonectomy had the operation on the

\section{TABLE 3. The probable etiology of bronchiectasis}

\begin{tabular}{lc}
\hline Etiology & $\mathbf{n}(\%)$ \\
\hline Postobstructive pneumonitis & $34(23.7 \%)$ \\
Pneumonia & $22(15.3 \%)$ \\
Tuberculosis & $22(15.3 \%)$ \\
Childhood infections & $19(13.2 \%)$ \\
Foreign body aspiration & $4(2.7 \%)$ \\
Pulmonary sequestration & $2(1.4 \%)$ \\
Unknown etiology & $40(27.9 \%)$ \\
\hline
\end{tabular}


TABLE 4. The operations carried out

\begin{tabular}{lcc}
\hline Operations & n & \% \\
\hline Lobectomy & 82 & 55.4 \\
Segmentectomy & 17 & 11.4 \\
Basal segmentectomy & 16 & 10.8 \\
Pneumonectomy & 12 & 8.3 \\
Bilobectomy & 7 & 4.7 \\
Basal segmentectomy + lingulectomy & 5 & 3.3 \\
Basal segmentectomy + middle lobectomy & 4 & 2.7 \\
Rethoracotomy owing to complications & 3 & 2.0 \\
Wedge resection & 2 & 1.3 \\
\hline
\end{tabular}

left side, and 4 on the right. Complete resection of all bronchiectasis was done in $118(82.5 \%)$ patients. Sixteen of $122(13.1 \%)$ patients with unilateral involvement had incomplete resections. In contrast, 9 of $11(81.8 \%)$ patients with bilateral involvement had incomplete resections. Postoperative HRCT was used to control whether the resection was complete or not. Complete resection is defined as an anatomic resection of all affected segments. Incomplete resection was noticed in 25 patients. Sixteen of the patients had unilateral bronchiectasis. Wedge resection was performed in 6 and segmentectomy was performed in 10 of these patients. Nine of the patients who had incomplete resection had bilateral bronchiectasis. Two of these patients died. Another 2 patients did not undergo a second operation because of their reasonably good outcomes. Five other patients refused a second operation although the second operation was planned. We consider that our slightly high incomplete resection rate is the result of our performing smaller than required resections in wedge resection and segmentectomy operations.

Complications occurred in 33 (23.0\%) patients (Table 5). Eleven patients had atelectasis postoperatively and were treated successfully with aspiration of the secretion through a fiberoptic bronchoscope. Seven of these 11 patients required repeated bronchoscopic treatments for the management of atelectasis. In 7 patients there was an air leak lasting more than 2 weeks, and in 1 of these patients empyema developed eventually. Six of 7 patients were treated with

TABLE 5. Complications after surgery for bronchiectasis

\begin{tabular}{lrr}
\hline Complications & n & $\%$ \\
\hline Atelectasis & 11 & 7.6 \\
Persistent air leak more than 15 days & 7 & 4.8 \\
Empyema & 5 & 3.4 \\
Supraventricular arrhythmias & 3 & 2.0 \\
Pneumonia & 3 & 2.0 \\
Postoperative hemorrhage & 2 & 1.3 \\
Respiratory insufficiency & 2 & 1.3 \\
Total & 33 & 23.0 \\
\hline
\end{tabular}

negative suction applied to their chest tubes, and 1 patient required surgical reintervention for closure of the fistula and myoplastic procedures. Five patients had empyema. They had tube thoracostomy and were successfully treated medically. Three patients had severe supraventricular arrhythmias, all controlled pharmacologically. Three patients had pneumonia and they were successfully treated medically. Two patients had postoperative hemorrhage requiring reexploration. During re-exploration, hemorrhage from the bronchial artery was observed in 1 patient, whereas the exact locus of hemorrhage could not be found in the other. Two patients had respiratory insufficiency that required mechanical ventilation. Mortality was seen in $2(1.3 \%)$ patients, caused by respiratory failure.

Follow-up was complete in 133 (93.0\%) patients with a mean of 4.2 years (range 6 months-12 years). One hundred one $(75.9 \%)$ patients were free of symptoms after surgical treatment, symptoms were improved in $21(15.7 \%)$, and were unchanged or worse in $11(8.2 \%)$. Of the patients who underwent complete resection, $98.3 \%$ benefited from the operation and, of those who underwent incomplete resection, only $12.1 \%$ benefited. The logistic regression analysis showed that tuberculosis history and incomplete resection were independent predictors of the no improvement group. Moreover, lack of preoperative bronchoscopy, a forced expiratory volume in 1 second of less than $60 \%$ of the predicted value, tuberculosis history, and incomplete resection were independent predictors of postoperative complications (Tables 6 and 7).

\section{Discussion}

There are many causes of bronchiectasis. Recurrent pulmonary infection during childhood is an important factor in the etiology. Similar to other series, most of our patients had recurrent infections in their histories, ${ }^{3,5,8}$ and most had histories of insufficient medication for pulmonary infection. Bronchial obstruction by foreign bodies is another important etiologic factor in children. In developing countries,

TABLE 6. Relation between clinical variables and postoperative complications

\begin{tabular}{lccc}
\hline Variables & OR & 95\% Cl & P value \\
\hline Gender (male:female) & 0.584 & $0.248-1.376$ & .219 \\
Age $(<17: \geq 17$ ys) & 1.926 & $0.728-5.108$ & .186 \\
Symptoms duration $(<12: \geq 12 \mathrm{~m})$ & 1.256 & $0.465-3.392$ & .653 \\
Positive culture (present:absent) & 1.025 & $0.466-2.253$ & .951 \\
Bronchoscopy (present:absent) & 0.084 & $0.032-0.220$ & .000 \\
Bronchiectasis type (saccular:others) & 0.964 & $0.441-2.105$ & .927 \\
FEV (value $<60 \%: \geq 60$ predicted) & 0.164 & $0.059-0.458$ & .001 \\
Type of resection & 0.031 & $0.010-0.096$ & .000 \\
(complete:incomplete) & & &
\end{tabular}

$\overline{O R}$, Odds ratio; $\mathrm{Cl}$, confidence interval; $F E V_{1}$, forced expiratory volume in 1 second. 
TABLE 7. Relations between clinical variables and operative results

\begin{tabular}{lccc}
\hline Variables & OR & 95\% Cl & $P$ value \\
\hline Gender (male:female) & 0.905 & $0.251-1.376$ & .878 \\
Age $(<17: \geq 17 \mathrm{y})$ & 3.191 & $0.392-25.966$ & .278 \\
Symptoms duration $(<12: \geq 12 \mathrm{mo})$ & 2.039 & $0.248-16.792$ & .508 \\
Positive culture (present:absent) & 2.590 & $0.720-9.313$ & .145 \\
Bronchoscopy (present:absent) & 0.337 & $0.090-1.258$ & .105 \\
Bronchiectasis type (saccular:others) & 1.457 & $0.422-5.027$ & .551 \\
FEV ${ }_{1}$ (value $<60 \%: \geq 60$ predicted) & 0.399 & $0.096-1.661$ & .207 \\
Type of resection & 0.031 & $0.006-0.155$ & .000 \\
(complete:incomplete) & & & \\
\hline
\end{tabular}

$O R$, Odds ratio; $\mathrm{Cl}$, confidence interval; $F E V_{1}$, forced expiratory volume in 1 second.

tuberculosis is still one of the most important causes of bronchiectasis. Bronchiectasis commonly develops between 1 and 3 months after the initial infection and usually in the same pulmonary region. ${ }^{9,10}$ This complication can be found in up to $11 \%$ of patients with tuberculosis. In a previous series, $84.2 \%$ of 120 patients, followed up because of bronchiectasis, had a history of tuberculosis. ${ }^{11,12}$ In Turkey, there is still a high incidence of pulmonary tuberculosis; hence, a significant number of our patients $(22 ; 15.3 \%)$ had a history of tuberculosis. In our study, tuberculosis history was an independent predictor of postoperative complication and operative result. Chronic inflammation and advanced pleural adhesions are commonly encountered in patients with a history of tuberculosis. We considered that this may be the reason for increased postoperative complications. Therefore, it is essential to soundly treat tuberculosis to prevent postinfectious bronchiectasis.

The presumptive diagnosis of bronchiectasis is still prompted by clinical findings. Chest radiographic findings that are suggestive but nondiagnostic of bronchiectasis include stranding, cystic lesions, volume loss with crowding of vessels, air-fluid levels and honeycombing, and areas of infiltrates and atelectasis. ${ }^{5} \mathrm{HRCT}$ is a modality of choice in the diagnosis of bronchiectasis, with only a $2 \%$ false negative and a $1 \%$ false positive rate. ${ }^{13}$ HRCT has now largely supplanted bronchography as a definite diagnosis and has become the best tool for the diagnosis of bronchiectasis. ${ }^{5}$

The standard of care for patients with bronchiectasis includes treatment of underlying conditions, management of infectious exacerbations, and bronchial hygiene. ${ }^{14}$ Preoperative bronchoscopic examinations were performed in 117 $(81.8 \%)$ of our patients as an adjuvant diagnostic method, to rule out benign or malignant bronchial obstruction, and for localization, collection of samples for microbiology, and bronchial toilet. We consider it essential in the preoperative evaluation and tracheobronchial cleaning of all patients. In our study, patients in whom bronchoscopy was not performed had a significantly higher rate of postoperative complications. Postoperative complications developed in 9 patients who did not receive bronchoscopic treatment. These complications were as follows: atelectasis in 7 patients, pneumonia in 1 patient, and respiratory insufficiency in 1 patient. Therefore, it should be routinely used preoperatively.

Pulmonary function studies were performed in 134 $(93.7 \%)$ of our patients. As was the case with other series, ${ }^{15,16}$ we found a normal ventilatory pattern in the majority of patients $(79.1 \%)$. The remainder showed a mixed or obstructive ventilatory pattern. In 19 (14.1\%) patients with low forced expiratory volume in 1 second $(<60 \%$ of the predicted value), the postoperative complication rate was significantly high. This indicated that surgery should be delayed in cases of severe inflammation until adequate control has been achieved. In addition, the preoperative treatment should include reducing airway obstruction and eliminating microorganisms from the lower respiratory tract, which consists of antimicrobial therapy, postural physiotherapy, bronchodilators, and corticosteroids. ${ }^{5}$

The goals of surgical therapy for bronchiectasis are to improve the quality of life for those patients in whom medical treatment has failed and to resolve complications such as empyema, severe or recurrent hemoptysis, and lung abscess. ${ }^{5,17}$ Complete and anatomic resection should be done with preservation of as much lung function as possible to avoid cardiorespiratory limitation. ${ }^{18}$ It was reported that the symptoms persisted when incomplete resection was carried out. ${ }^{19}$ We performed complete resection in $82.5 \%$ of our patients, and preoperative symptoms resolved completely in $75.9 \%$ and improved in $15.7 \%$ (ie, $91.6 \%$ benefited from the operation). In the light of these findings, we suggest that complete resection should be performed for the surgical treatment of bronchiectasis and that incomplete resection should only be used for the palliative treatment of certain life-threatening symptoms.

Postoperative complications were observed in $11 \%$ of patients who underwent complete resection and in $80 \%$ of those who underwent incomplete resection. When suggestive lung regions are not excised, with the aim of sparing as much lung tissue as possible, more postoperative complications occur and a second operation that carries a higher morbidity and mortality might be required to remove the residual diseased tissues. ${ }^{20}$ Therefore, we suggest that, during intraoperative examinations, if suggestive areas that could not be determined by radiologic examination are present, these parenchymal areas should be resected to perform complete resection and to decrease relapse rates.

Surgical treatment is usually offered only when the diseased area is well localized and restricted to one or several segments within the same lobe. Surgery in multiple segments on different lobes is technically more difficult, resulting in higher morbidity and mortality. ${ }^{21}$ In these patients 
resection is recommended only for severe life-threatening hemoptysis or infection-related complications. ${ }^{5,22}$ However, pulmonary resection is indicated early in patients with segmentary bronchiectasis, before other portions of the lung become grossly diseased. In 5 of our patients, resection of the basal segments of the left lower lobe without the apical segment plus lingulectomy was performed. In addition, in 4 of our patients, right middle lobectomy, protecting the apical segment of the right lower lobe, along with basal segmentectomy was performed. Our purpose was to protect as much pulmonary function as possible, with the aim of removing only the affected areas of different lobes. Of these patients, 1 had complications that required rethoracotomy for hemothorax. In another patient, pneumonia developed, which responded to conservative treatment. Yuncu and associates $^{23}$ observed no morbidity in 3 patients who underwent multiple-segment resection. Schneiter and colleagues ${ }^{21}$ reported minor complications requiring medical therapy in 3 of 23 patients with bronchiectasis affecting at least one segment in two different lobes.

Sixteen patients underwent basal segmentectomy with preservation of the superior segment. As recommended, ${ }^{8}$ we tried to protect the anatomic structure of the superior segment in cases of bronchiectasis of the lower lobe when the superior segment of this lobe was normal. In such patients, the superior segment had undergone a compensatory increase in volume and the affected basal segments had become small. Thus, the functional value of the superior segment was similar to that of the lower lobe. ${ }^{23}$ Patients with an uninvolved apical segment were found to have better spirometric values than those with more extensive disease. ${ }^{24}$ Atelectasis of the preserved superior segment of the lower lobe occurred in 1 of 16 patients who underwent basal segmentectomy, and it could be treated with fiberoptic bronchoscopic aspiration. In another patient, a postoperative air leak lasting 1 week was observed, but the negative section was treated conservatively.

Bronchiectasis is an inflammatory disease of the lungs, and the risk of postoperative empyema developing is higher than in other cases. Empyema, on the other hand, is a risk factor for bronchopulmonary fistula. ${ }^{20}$ Therefore, we suggest reinforcement of the bronchial stump in all patients. Additionally, to avoid empyema, we recommend postoperative bacterial culture of thoracic effusion if the remaining lung shows signs of persisting inflammation. Sputum retention is common because patients with this disease might have problems with ciliary motion and postoperative expectoration, which would be easily disrupted. ${ }^{8}$ In our series, respiratory physiotherapy was reinitiated on the first postoperative day and continued for 2 weeks after discharge. We used bronchoscopy for sputum aspiration during the early postoperative period if physiotherapy was not effective. Virtually all patients had specific or large-spectrum intra- venous antibiotic therapy for 1 week. The rate of complications in the current literature varies between $9.4 \%$ and $24.6 \% .^{8}$ In our series, the rate was $23.0 \%$, in agreement with the literature. Mortality ranges from $0 \%$ to $8.3 \%$ in the literature and mortality in our series was $1.3 \%$. There was no operative mortality in our series.

\section{Conclusions}

Tuberculosis history and incomplete resection were independent predictors both of postoperative complications and of worse operative results. A lack of preoperative bronchoscopy and low forced expiratory volume in 1 second were independent predictors of postoperative complications. Patients should be well prepared during the preoperative period with regard to infection to minimize postoperative complications. Surgical resection aimed to minimize the number of resected segments, without compromising the objective of eliminating the disease. Surgery for multiple segments on different lobes should be performed whenever possible. When necessary, surgical treatment of bronchiectasis can be performed with acceptable morbidity and low mortality according to our results.

\section{References}

1. Balkanli K, Genc O, Dakak M, Gurkok S, Gozubuyuk A, Caylak H, et al. Surgical management of bronchiectasis: analysis and short-term results in 238 patients. Eur J Cardiothorac Surg. 2003;24:699-702.

2. Miller JI. Bacterial infections of the lungs and bronchial compressive disorders. In: Shields TW, editor. General thoracic surgery. 5th ed. Philadelphia: Lippincott Williams \& Wilkins; 2000. pp 1048-51.

3. Prieto D, Bernardo J, Matos MJ, Eugenio L, Antunes M. Surgery for bronchiectasis. Eur J Cardiothorac Surg. 2001;20:19-23.

4. Ashour M, Al-Kattan K, Rafay MA, Saja KF, Hajjar W, Al-Fraye AR. Current surgical therapy for bronchiectasis. World J Surg. 1999;23:1096-104.

5. Agasthian T, Deschamps C, Trastek VF, Allen MS, Pairolero PC. Surgical management of bronchiectasis. Ann Thorac Surg. 1996;62:976-8.

6. Ellis DA, Thornley PE, Wightman AJ, Walker M, Chalmers J, Crofton JW. Present outlook in bronchiectasis: clinical and social study and review of factors influencing prognosis. Thorax. 1981;36:659-64.

7. Eren S, Eren MN, Balcı AE. Pneumonectomy in children for destroyed lung and the long-term consequences. $J$ Thorac Cardiovasc Surg. 2003;126:574-81.

8. Fujimoto T, Hillejan L, Stamatis G. Current strategy for surgical management of bronchiectasis. Ann Thorac Surg. 2001;72:1711-5.

9. Karakoc GB, Yilmaz M, Altintas DU, Kendirli SG. Bronchiectasis: still a problem. Pediatr Pulmonol. 2001;32:175-8.

10. Haciibrahimoglu G, Fazlioglu M, Olcmen A, Gurses A, Bedirhan MA. Surgical management of childhood bronchiectasis due to infectious disease. J Thorac Cardiovasc Surg. 2004;127:1361-5.

11. Bogossian M, Santoro IL, Jamnik S, Romaldini H. Bronchiectasis: a study of 314 cases tuberculosis x non-tuberculosis. J Pneumol.1998; 24:11-16.

12. Marostica PJ, Fischer GB. Non-cystic-fibrosis bronchiectasis: a perspective from South America. Paediatr Respir Rev. 2006;7:275-80.

13. Young K, Aspestrand F, Kolbenstvedt A. High resolution CT and bronchography in the assessment of bronchiectasis. Acta Radiol. 1991; 32:439-41.

14. Barker AF. Bronchiectasis. N Engl J Med. 2002;346:1383-93.

15. Thevenet F, Gamondes JP, Cordier JF, Tekinel O, Adeleine P, Bodzongo $\mathrm{D}$, et al. Surgery for bronchiectasis. Operative indications and results. 48 observations. Rev Mal Respir.1993;10:245-50.

16. Cherniack NS, Carton RW. Factors associated with respiratory insufficiency in bronchiectasis. Am J Med. 1966;41:562-71. 
17. Annest LS, Kratz JM, Crawford FA Jr. Current results of treatment of bronchiectasis. J Thorac Cardiovasc Surg. 1982;83:546-50.

18. Laros CD, Van den Bosch JM, Westermann CJ, Bergstein PG, Vanderschueren RG, Knaepen PJ. Resection of more than 10 lung segments. A 30-year survey of 30 bronchiectatic patients. J Thorac Cardiovasc Surg. 1988;95:119-23.

19. Campbell DN, Lilly JR. The changing spectrum of pulmonary operations in infants and children. J Thorac Cardiovasc Surg. 1982;83: 680-5.

20. Sirmali M, Karasu S, Turut H, Gezer S, Kaya S, Tastepe I, et al. Surgical management of bronchiectasis in childhood. Eur J Cardiothorac Surg. 2006 Nov 18; [Epub ahead of print].
21. Schneiter D, Meyer N, Lardinois D, Korom S, Kestenholz P, Weder W. Surgery for non- localized bronchiectasis. Br J Surg. 2005;92: 836-9.

22. Kutlay H, Cangir AK, Enon S, Sahin E, Akal M, Gungor A, et al. Surgical treatment in bronchiectasis: analysis of 166 patients. Eur J Cardiothorac Surg. 2002;21:634-7.

23. Yuncu G, Ceylan KC, Sevinc S, Ucvet A, Kaya SO, Kiter G, et al. Functional results of surgical treatment of bronchiectasis in a developing country. Arch Bronconeumol2006;42:183-8.

24. Ashour M, Al-Kattan KM, Jain SK, Al-Majed S, Al-Kassimi F, Mobaireek A, et al. Surgery for unilateral bronchiectasis: results and prognostic factors. Tuber Lung Dis.1996;77:168-72.

To assure fairness to authors submitting work for consideration in The Journal of Thoracic and Cardiovascular Surgery, a mechanism exists for managing conflicts of interest. The editor and each of the section editors complete a "Conflict of Interest" form that identifies any and all relationships with commercial and other academic entities. When the editor has a potential conflict because of a relationship with another entity or author, the editor appoints an alternate editor from among the section editors or editorial board members who assumes the entire responsibility for final decisions on the manuscript in question. The editor does not read the reviews that are submitted nor engage in discussing the manuscript prior to the final decision. When the conflict of interest involves a section editor, a "guest section editor" is appointed who fills the role normally played by the conflicted section editor. All members of the editorial board and reviewers are asked to indicate any conflict of interest when they agree to review a manuscript. 